\title{
Low Loss Microwave Ceramic and other Microwave Dielectric Materials for Beam Physics Applications
}

\author{
Alexei D. Kanareykin ${ }^{\bowtie}$ \\ Euclid Techlabs LLC \\ 5900 Harper Rd, Solon, OH 44139, USA \\ 凶alexkan@euclidtechlabs.com
}

\begin{abstract}
Introduction. Relativistic, high intensity and small emittance electron bunches are the basis of a future linear collider and free electron laser projects. Drive beam generation in a wakefield structure employing for power extraction and acceleration low loss dielectrics like microwave ceramics, fused silica and Chemical Vapor Deposition (CVD) diamond were considered.

Objective. We report here our experimental testing of a ceramic material with extremely low loss tangent at $\mathrm{GHz}$ frequency ranges allowing the realization of high efficiency wakefield acceleration. We also present Barium Strontium Titanium oxides (BST) ferroelectric material, which is a critical tuning element of the $400 \mathrm{MHz}$ superconducting radiofrequency (RF) tuner developed and tested by the CERN/Euclid Techlabs collaboration. The materials discussed here also include quartz and CVD diamonds that are capable of supporting the high RF electric fields generated by electron beams or pulsed high power microwaves. These materials have been optimized or specially designed for accelerator applications.
\end{abstract}

Materials and methods. The ceramic materials for accelerators, commonly used for the dielectric based accelerating structures, have to withstand high gradient accelerating fields, and prevent potential charging by electron beams. Correspondingly, the ceramic materials, fused silica and CVD diamond were tested with high power wakefield accelerating structures at Argonne Wakefield Accelerator of Argonne National Laboratory. Some of the presented here ceramic materials were tested at X-band $11.4 \mathrm{GHz}$ magnicon high power source.

Results. Low loss microwave ceramics, fused silica, and CVD diamonds have been considered as materials for dielectric based accelerating structures to study of the physical limitations encountered driving $>100 \mathrm{MV} / \mathrm{m}$ at microwave and $\sim \mathrm{GV} / \mathrm{m}$ at THz frequencies in a dielectric based wakefield accelerator. Various ceramic compositions were high power and electron beam tested at X-band $11.4 \mathrm{GHz}$ magnicon power source and Argonne Wakefield Accelerator correspondingly. Special attention was paid to the CVD diamond cylindrical Ka-band $35 \mathrm{GHz}$ wakefield structure development. Finally, the dielectric based structure tuning was demonstrated by varying the permittivity of the BST ferroelectric layer by temperature changes and by applying an external direct current electric field across the ferroelectric. This allows us to control the effective dielectric constant of the composite system and therefore, to control the structure frequency during operation. The same type of ferroelectric material was used for the Ferroelectric Fast Reactive tuner (FE-FRT) development. In a world first, CERN has tested the prototype FE-FRT with a superconducting cavity, and frequency tuning has been successfully demonstrated.

Conclusion. Recent results on the development and experimental testing of advanced dielectric materials for accelerator applications are presented. Low loss microwave ceramics, quartz and CVD diamond are considered. We presented our experimental results on wakefield generation in microwave frequency ranges with the dielectric based accelerating structures. Special attention was paid to the experimental results on high power testing at X-band of the externally powered dielectric based components. Finally, we present here first experimental demonstration of ferroelectric tunable microwave ceramic for accelerator application, which includes both tunable dielectric wakefield accelerating structure and ferroelectric based fast high power tuner for superconducting cavities. The experimental results presented here are critical for the advanced dielectric wakefield accelerating structures and other components development intended for the future linear collider projects.

Key words: microwave, dielectric, ceramic, diamond, ferroelectric, accelerator 
For citation: Kanareykin A. D. Low Loss Microwave Ceramic and other Microwave Dielectric Materials for Beam Physics Applications. Journal of the Russian Universities. Radioelectronics. 2019, vol. 22, no. 4, pp. 66-74. doi: 10.32603/1993-8985-2019-22-4-66-74

Acknowledgements. The author would like to thank the research staff of Euclid Techlabs LLC, Solon OH, USA, Ceramics Ltd. of St. Petersburg, Russia and Argonne Wakefield Accelerator of Argonne National Laboratory, Chicago IL, USA for their support and assistance with this paper.

Conflict of interest. The author declares no conflict of interest.

Submitted 15.08.2019; accepted 10.09.2019; published online 27.09.2019

Introduction. A linear collider concept based on drive beam generation from an radiofrequency (RF) photoinjector and employing dielectric structures [1] for power extraction and acceleration has been proposed [2]. The collider is based on a modular design with each module providing $100 \mathrm{GeV}$ net acceleration. High frequency $(20 \mathrm{GHz}) \mathrm{RF}$ power extracted from the drive beam using a low impedance dielectric structure is used to power the main linacs, which are based on high impedance high gradient dielectric loaded accelerating structures $[2,3]$. Dielectric structures have been shown to sustain high fields for short RF pulse lengths and can be used as RF power generation devices [3-5]. In this paper, we present a survey of experimental testing of microwave materials with high-current electron beams and high power microwave sources intended for use in dielectric based short pulse linear collider designs [2].

Low loss microwave ceramics, fused silica, and Chemical Vapor Deposition (CVD) diamonds have been considered as materials for dielectric based accelerating structures to study of the physical limitations encountered driving $>100 \mathrm{MV} / \mathrm{m}$ at microwave [3-5] and $>\mathrm{GV} / \mathrm{m}$ at $\mathrm{THz}$ frequencies in a dielectric based wakefield accelerator [6]. Advanced dielectric materials can sustain high surface fields and high pulsed power in the $\mathrm{GHz}$ frequency range [4]. A $100 \mathrm{MV} / \mathrm{m}$ gradient has been demonstrated with an $\mathrm{X}$-band standing wave resonator $[3,4]$. THz radiation has been generated by a short $\sim 10 \mathrm{GV} / \mathrm{m}$ pulse within a $100 \mu \mathrm{m}$ diameter dielectric fiber [6]. The electrical and mechanical properties of diamond make it an ideal candidate material for use in dielectric RF structures: high breakdown voltage, extremely low dielectric losses and the highest thermal conductivity coefficient available for removing waste heat from the device [7-9].

Recently developed nonlinear "smart" materials like Barium Strontium Titanium (BST) based low loss ferroelectrics are used as key elements in RF tuning and phase shifting components to provide fast electronic control $[10,11]$. The frequency of a metallic accelerating structure is defined by its geometry. Dielectric loaded accelerator (DLA) structures, on the other hand, have another important parameter that determines the frequency spectrum - the dielectric constant of the loading material. A new technique that allows control of the dielectric constant (and consequently the frequency spectrum) for dielectric waveguides by incorporating ferroelectric layer is considered. It should be noticed that ferroelectric materials are also is a base for newly developed accelerator components that allow coupling adjustment and control of power consumption during accelerating cavity filling and are under study for potential Energy Recovery Linac (ERL), International Linear Collider (ILC) and Proton Improvement Plan-II (PIP-II) applications [12, 13].

Methods. Dielectric loaded accelerator structures using low-loss microwave ceramics and excited by a high current electron beam or an external high frequency high power RF source have been under extensive study [1, 3-6]. The basic wakefield RF structure is very simple - a cylindrical, dielectric loaded waveguide with an axial vacuum channel is inserted into a conductive sleeve. A high charge, (typically $20 \ldots 40 \mathrm{nC}$ ), short, (1-4 mm) electron drive beam generates TM0n mode electromagnetic Cherenkov radiation (wakefields) while propagating down the vacuum channel. Following at a delay adjusted to catch the accelerating phase of the wakefield is a second electron (witness) beam. The witness beam is accelerated to high energy directly by the wakefield produced by the drive beam in the collinear accelerating method [1, 2-6]. In a twobeam-acceleration method, a low-energy, high-current particle beam is passed through a deceleration section, where the power from the beam is partially transferred to wakefields. With a properly designed RF output coupler, the power can be extracted to an output waveguide, and used to accelerate a high-energy low- 
current beam $[3,4,14]$. A series of proof of principle experiments have been successfully performed in microwave frequency range at the Argonne Advanced Accelerator Test Facility and Argonne Wakefield Accelerator $[1,3-5]$. THz range wakefields have been generated by the UCLA-SLAC collaboration [6].

The advantages and potential problems of using dielectric for loading an accelerating structure are discussed in the references $[3,4,15]$ and are only summarized here. The advantages are:

- simplicity of fabrication: the device is simply a tube of dielectric surrounded by a conducting cylinder that is a great advantage for high frequency structures compared to conventional structures, where extremely tight fabrication tolerances are required;

- the relatively small diameter of these devices also facilitates placement of quadrupole lenses around the structures useful for suppressing beam break up effects;

- dielectrics can potentially exhibit high breakdown thresholds relative to copper and high shunt impedance;

- reduced sensitivity to the single bunch beam break-up (BBU) instability;

- easy parasitic mode damping $[3,4,15]$.

Potential challenges of using dielectric materials in a high power RF environment are breakdown and thermal heating, challenges that are shared with allmetal conventional accelerating structures.

Results and discussion. The first dielectric wakefield acceleration was demonstrated at the Argonne Accelerator Test Facility at Argonne National Laboratory (ANL) [1]. This accelerator provided a beamline for these pioneer wakefield acceleration experiments. The charge per bunch could be varied between 1 and $5 \mathrm{nC}$, and part of this bunch was subsequently intercepted by a graphite target to form a second beam. Materials used in this proof-of principal experiment were polystyrene, steatite and nylon that were acceptable at that time for the demonstrated low wakefields magnitudes $<1 \mathrm{MV} / \mathrm{m}$ [1].

The Argonne Wakefield Accelerator (AWA) facility was built in the early 1990s, having as its main objective the generation of high charge electron beams for wakefield acceleration and RF power generation [3-4]. A single cell photocathode RF gun was used to produce high charge drive electron bunches of charge up to $100 \mathrm{nC}$ accelerated by two linac tanks to an energy of $15 \mathrm{MeV}$; a second multi-cell photocathode RF gun was used to generate $4 \mathrm{MeV}$ witness bunches. The two beamlines - drive beam and witness beam - were connected by a combining section that provided drive and witness beams that could be configured as both a collinear wakefield accelerator and two beam accelerator [3-4, 14].

Several materials have been beam tested at the AWA facility to demonstrate dielectric based acceleration. An X-band dielectric loaded accelerator has been developed [15], and several standing-wave DLA structures have been tested under high gradients up to $100 \mathrm{MV} / \mathrm{m}[3,4]$. Recently lower power loss and higher acceleration efficiency multilayer DL structures were proposed [16] and bench tested [17]; an enhanced transformer ratio was shown in a ramped bunch train test in a DLA structure [18]. Different materials such as cordierite $(\varepsilon=4.7)$, forsterite $(\varepsilon=6.3)$, magnesium calcium titanium oxides (MCT) $(\varepsilon=20)$, alumina $(\varepsilon=9.8)$ and quartz $(\varepsilon=3.8)$ have been used for different applications, which provided useful experience in choosing dielectric materials and testing RF breakdown thresholds.

Dielectric loaded wakefield structures have potential to be used as high gradient accelerator components, and $15 \mathrm{MeV}$ drive beam measurements of the ceramic and quartz materials are summarized in Table 1 of this paper. The table is limited to the work of the AWA group and contributions from their collaborators. After the AWA demonstrated its capability of producing $>100 \mathrm{nC}$ per single bunch, attempts were made to achieve high accelerating gradients in several short standing-wave structures using wakefields from a high charge beam [3, 4]. Quartz and cordierite structures have been recently beam tested, with dielectric constants of 3.75 and 4.76, respectively. The AWA group has been able to gradually increase the gradient in dielectric wakefield devices from $20 \mathrm{MV} / \mathrm{m}$ a few years ago to the current $100 \mathrm{MV} / \mathrm{m}$. The results show no observed breakdown [3-4], Table 1.

A method of power extraction has to be provided for a two-beam accelerator design $[3,4,14]$. As part of the two-beam accelerator program at AWA, a $7.8 \mathrm{GHz} \mathrm{DL}$ power extractor has been designed and tested with an intense electron beam. $30 \mathrm{MW}$ of power has been generated in single bunch experiments and $44 \mathrm{MW}$ in tests using a train of four bunches [19]. Furthermore, a $26 \mathrm{GHz}$ DL power extractor has been designed and demonstrated in 2009, and $30 \mathrm{MW}$ output power has been reached in a 10 
Table 1. Ceramic microwave materials that have been utilized for the dielectric loaded accelerating (DLA) structure development

\begin{tabular}{|c|c|c|c|c|}
\hline Dielectric & $\varepsilon$ & $\tan \delta, 10 \mathrm{GHz}$ & Frequency, GHz & Experiment, reference \\
\hline Quartz & 3.8 & $<1 \cdot 10^{-4}$ & 11.4 & High Gradient Standing Wave $[3,4]$ \\
\hline Cordierite & 4.7 & $<1.5 \cdot 10^{-4}$ & $\begin{array}{c}7.8 \\
8.6 \\
21 \\
11.4 / 30\end{array}$ & $\begin{array}{c}\text { 7.8 GHz TBA Power Extractor [14] } \\
\text { High Gradient Standing Wave [3, 4] } \\
21 \mathrm{GHz} \text { DL Power Extractor [19] } \\
\text { Two Channel High Transformer Ratio [23] }\end{array}$ \\
\hline Diamond & 5.7 & $<1 \cdot 10^{-4}$ & $26-35$ & High Gradient Standing Wave [5, 9] \\
\hline Forsterite & 6.3 & $<2 \cdot 10^{-4}$ & $\begin{array}{c}11.4 \\
26\end{array}$ & $\begin{array}{c}\text { Traveling Wave DLA [13] } \\
26 \text { GHz DL Power Extractor [18] }\end{array}$ \\
\hline Alumina & 9.8 & $<1 \cdot 10^{-4}$ & 11.4 & Dual Layer DLA (outer layer) [16] \\
\hline $\begin{array}{l}\mathrm{MgTiO}_{3}- \\
\mathrm{Mg}_{2} \mathrm{TiO}_{4}\end{array}$ & 16 & $<1.2 \cdot 10^{-4}$ & 13.6 & Collinear High Transformer Ratio [17] \\
\hline $\begin{array}{c}\mathrm{MCT} \\
(\mathrm{Mg}, \mathrm{Ca}) \mathrm{TiO}_{3}\end{array}$ & 20 & $<2 \cdot 10^{-4}$ & 7.8 & Two Beam DLA Accelerator [12] \\
\hline $\mathrm{BaTi}_{4} \mathrm{O}_{9}$ & 37 & $<3 \cdot 10^{-4}$ & 11.4 & Dual Layer DLA (inner layer) [16] \\
\hline $\begin{array}{l}\mathrm{CaTiO}_{3}- \\
\mathrm{LaAlO}_{3}\end{array}$ & 38.1 & $<5 \cdot 10^{-4}$ & $1 \ldots 30$ (multimode) & Multimode DLA bunch train generation [22] \\
\hline
\end{tabular}

ns pulse [20]. No breakdown phenomena have been detected with this set of experiments either. The $21 \mathrm{GHz}$ DL power extractor has been tested previously by Duly Research, with the results reported in [21].

A joint ANL/NRL/Euclid program in 2005-2015 allowed to investigate $\mathrm{X}$-band DLA structures, using high-power $11.424 \mathrm{GHz}$ RF from the NRL Magnicon Facility $[22,23]$. The X-band NRL Magnicon can provide up to $20 \mathrm{MW}$ with a $200 \mathrm{~ns}$ pulse length from either of its two output arms to test DLA designs and in particular to determine the RF breakdown limits of the structure materials. The most recent test results for two DLA structures loaded with alumina and MCT have been reported in ref. [22]. No RF breakdown has been observed for up to $5 \mathrm{MW}$ of drive power (equivalent to $8 \mathrm{MV} / \mathrm{m}$ accelerating gradient), but multipactor was observed to absorb a large fraction of the incident microwave power. The latest experimental results on suppression of multipactor using a Titanium Nitride (TiN) coating on the inner surface of the dielectric have been reported in $[22,23]$. It was found that the MCT structure appears to be superior to the alumina structure as it loses less power throughout the tested range.

The DLA structure loading materials tested at the NRL magnicon are presented in Table 2. The most recently built design is the X-band clamped DLA structure made of quartz as the loading dielectric with an inner diameter of $3 \mathrm{~mm}$. This structure is designed to produce an $11.6 \mathrm{MV} / \mathrm{m}$ accelerating gradient at $10 \mathrm{MW}$ RF drive power. This structure was tested to an input power level of 16.5 MW with no sign of RF breakdown, for which a gradient of $15 \mathrm{MV} / \mathrm{m}$ can be inferred. The $10 \% \mathrm{RF}$ transmission

Table 2. Microwave dielectric materials that have been high power tested at the X-band NRL Magnicon Facility

\begin{tabular}{|c|c|c|c|c|}
\hline Dielectric & $\varepsilon$ & $\tan \delta$ & $\begin{array}{c}\text { Frequency, } \\
\mathrm{GHz}\end{array}$ & $\begin{array}{c}\text { Experiment, } \\
\text { reference }\end{array}$ \\
\hline Quartz & 3.8 & $<1 \cdot 10^{-4}$ & 11.4 & $\begin{array}{c}\text { Traveling } \\
\text { Wave 250 } \\
\text { ns RF Pulse } \\
{[21]}\end{array}$ \\
\hline Alumina & 9.8 & $<1 \cdot 10^{-4}$ & 11.4 & $\begin{array}{c}\text { Traveling } \\
\text { Wave 250 } \\
\text { ns RF Pulse } \\
{[20,21]} \\
\text { Dual Layer } \\
\text { DLA (outer } \\
\text { layer) [16] }\end{array}$ \\
\hline $\left.\mathrm{MCT}^{*} \mathrm{Ca}\right) \mathrm{TiO}_{3}$ & 20 & $<2 \cdot 10^{-4}$ & 11.4 & $\begin{array}{c}\text { Traveling } \\
\text { Wave 250 } \\
\text { ns RF Pulse } \\
{[21]}\end{array}$ \\
\hline $\mathrm{BaTi}_{4} \mathrm{O}_{9}$ & 37 & $<3 \cdot 10^{-4}$ & 11.4 & $\begin{array}{c}\text { Dual Layer } \\
\text { DLA (inner } \\
\text { layer) [16] }\end{array}$ \\
\hline
\end{tabular}


drop as the input power was increased up to $4 \mathrm{MW}$ reflects the onset of multipactor induced losses. This structure showed no sign of RF breakdown at the highest input powers applied [22].

Light reflects off the naturally smooth individual facets of diamond crystals comprising the polycrystalline aggregate.

It should be noticed that multiple arcing has been detected with initial high power tests. The RF power applied to the first MCT structure was stopped at 1.9 MW due to multiple arcing sites that appeared at a dielectric joint between the upstream end taper section and the uniform acceleration section resulting from the existence of a microscale vacuum gap in the dielectric joint [23]. Based on the continuity of electric flux, the local longitudinal electric field should be enhanced by $20(\varepsilon)$ times compared to the ideal (joint free) case. For this MCT DLA structure, the accelerating field is around $5.7 \mathrm{MV} / \mathrm{m}$ (10\% power reflection considered) at the upstream end taper when $1 \mathrm{MW}$ of incident power is applied. One can estimate that the electric field enhancement at the gap to be $100 \mathrm{MV} / \mathrm{m}$ for a $5.7 \mathrm{MV} / \mathrm{m}$ accelerating gradient. Interestingly, this indirectly shows that the MCT DLA structure handled $60 \ldots 100 \mathrm{MV} / \mathrm{m}$ electric fields for a $200 \ldots 250 \mathrm{~ns} \mathrm{RF}$ pulse without breakdown [23].

In order to eliminate dielectric joints, two new types of structures were developed that employ a single-section dielectric insert. One uses a coaxial RF coupler, and the other structure uses a clamped metal outer jacket to enclose a tapered dielectric insert. The X-band clamped DLA structure using quartz as the loading material has been fabricated and tested at NRL Magnicon Facility. As expected, this structure showed no signs of $\mathrm{RF}$ breakdown at the highest powers tested [22].

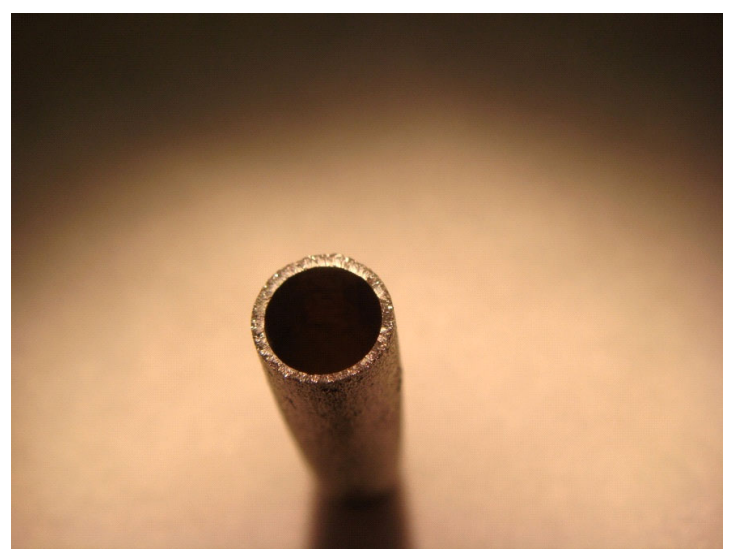

$a$
RF power attenuation is a critical problem in the development of dielectric loaded structures for particle acceleration. A Multilayer Dielectric Loaded Accelerating Structure (MDLA) is a possible approach for reducing the RF losses below those of a single layer device [17]. A MDLA based on the principle of Bragg reflection has been designed and constructed using alumina and $\mathrm{BaTi}_{4} \mathrm{O}_{9}$ low loss ceramic as loading materials (Tables 1,2). The cold test results are presented in [17]; beam and high power tests are planned in nearest future. $13.625 \mathrm{GHz}$ DLA structure was designed fabricated with $\mathrm{MgTiO}_{3}-\mathrm{Mg}_{2} \mathrm{TiO}_{4}$ ceramic loading $(\varepsilon=16)$ for the High Transformer Ratio experiment [18]. A multimode DLA structure $\left(\mathrm{CaTiO}_{3}-\mathrm{LaAlO}_{3}, \varepsilon=16\right)$ has been excited with the four-bunch train as well [24]. A two channel DLA based structure with enhanced transformer ratio and made of cordierite ceramic is currently being tested at AWA [25]. No breakdown or multipactoring were detected in these experiments.

Low-loss microwave ceramics and quartz are not the only materials that are being intensively studied as potential DLA loading. An alternative is to use polycrystalline artificial diamond produced by CVD [9], which shows promise for use in a high-gradient, DLA structure. It has a very high breakdown field up to $2 \mathrm{GV} / \mathrm{m}$ at DC field (no data available for RF frequencies yet), low loss tangent $\left(<10^{-4}\right.$ at $\left.10 \ldots 30 \mathrm{GHz}\right)$, and the highest known thermal conductivity $\left(2 \cdot 10^{3} \mathrm{~W} \cdot \mathrm{m}^{-1} \cdot \mathrm{K}^{-1}\right)[7-9]$.

Initially, a diamond based resonator loading was considered in [7]. In [8], a diamond-based rectangular DLA was discussed and all-metal and dielectric-based

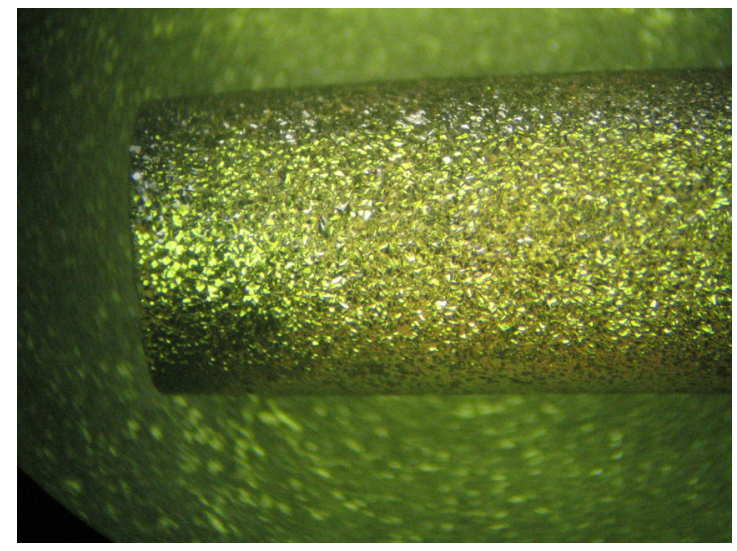

$b$

Fig. 1. Photographs of CVD diamond tube developed: top view ( $a$ ); axial view ( $b$ ) 
Известия вузов России. Радиоэлектроника. 2019. Т. 22, № 4 Journal of the Russian Universities. Radioelectronics. 2019, vol. 22, no. 4
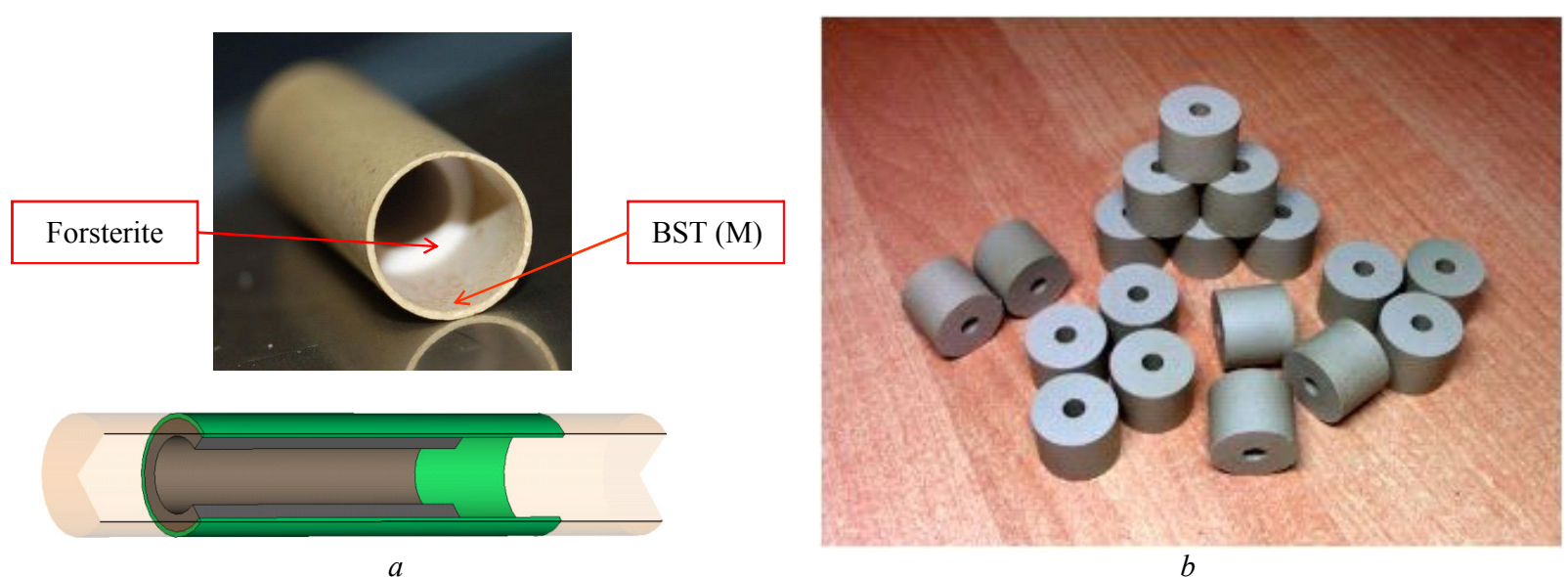

Fig. 2. The tunable DLA resonator with double layer loading: the nonlinear layer is made of BST(M) ferroelectric and the inner forsterite layer (light) inserted into the ferroelectric one (a); tunable ferroelectric elements for BST based superconducting RF (SRF) tuner prototypes (b)

accelerating structures were compared. The cylindrical diamond dielectric tubes that are manufactured via a relatively simple and inexpensive CVD process, plasma assisted CVD have been considered in [5, 9]. Our initial work was based on $100 \mu \mathrm{m}$ and $5 \mathrm{~mm}$ scale tubes with fundamental frequencies in the $0.1 \ldots 1.0 \mathrm{THz}$ range; promising results were obtained using the plasma assisted and hot-filament CVD process. The $5 \mathrm{~mm}$ inner diameter, $2.5 \mathrm{~cm}$ long and $500 \mu \mathrm{m}$ thick diamond tube has been fabricated for $35 \mathrm{GHz}$ structure and characterized with SEM, micro-Raman and micro-photoluminescence spectrum analysis. Fig. 1 shows the finished free standing diamond tube. Tube parameters are: $5 \mathrm{~mm}$ inner diameter, $2.5 \mathrm{~cm}$ long and $\sim 500 \mu \mathrm{m}$ thick.

The frequency of a metallic accelerating structure is defined by its geometry. DLA structures, on the other hand, have another important parameter that determines the frequency spectrum - the dielectric constant of the loading material. We have proposed a new technique that allows control of the dielectric constant (and consequently the frequency spectrum) for dielectric waveguides by incorporating ferroelectric layers. The most noteworthy feature of the tunable DLA is the replacement of a single ceramic by a composite of 2 layers as shown in Fig. 2, $a$. The inner layer is ceramic, with permittivity typically in the range of $4 \ldots 36[5,10]$. The outer layer is made of BST ferroelectric, placed between the ceramic layer and the copper sleeve. The DLA structure tuning is achieved by varying the permittivity of the ferroelectric layer by temperature changes or by applying an external DC electric field across the ferroelectric. This allows us to control the effective dielectric constant of the composite system and therefore, to control the structure frequency during operation. Table 3 presents materials that have been used for the tunable DLA fabrication. The details can be found in $[10,12]$, where the results show a very good linearity and sensitivity of the thermal tuning of this ferroelectric material: $\sim 14 \mathrm{MHz} / \mathrm{K}$ has been measured for the structures.

The BST solid solution can be synthesized in the form of polycrystalline ceramic layers and in bulk [12]. High dielectric breakdown strength, low gas permeability and simplicity of mechanical treatment make ferroelectric ceramics promising candidates for the loading material in accelerator tuning and switching devices [12]. Recent progress in the technology of BST(M) ferroelectrics was demonstrated in laboratory tests of fast switching: switching times $<1 \mathrm{~ns}$ were measured for small ferroelectric samples and $<30 \mathrm{~ns}$ for a high power L-band tuner model $[12,13]$. Other desired material properties are quite well known: high tunability and low dielectric losses are required. Consequently our work has focused on ferroelectric-dielectric composites containing inclu-

Table 3. Microwave dielectric and ferroelectric materials that have been used for the tunable DLA fabrication

\begin{tabular}{|c|c|c|c|c|}
\hline Dielectric & $\varepsilon$ & $\tan \delta$ & Frequency, GHz & Experiment, reference \\
\hline Forsterite & 6.3 & $<2 \cdot 10^{-4}$ & $18 \ldots 26$ & Tunable DLA [10,11] \\
\hline $\mathrm{BST} / \mathrm{MgO} / \mathrm{Mg}_{2} \mathrm{TiO}_{4}$ & $100 \ldots 500$ & $<1 \cdot 10^{-3}$ & $18 \ldots 26$ & Tunable DLA [10,11] \\
\hline
\end{tabular}



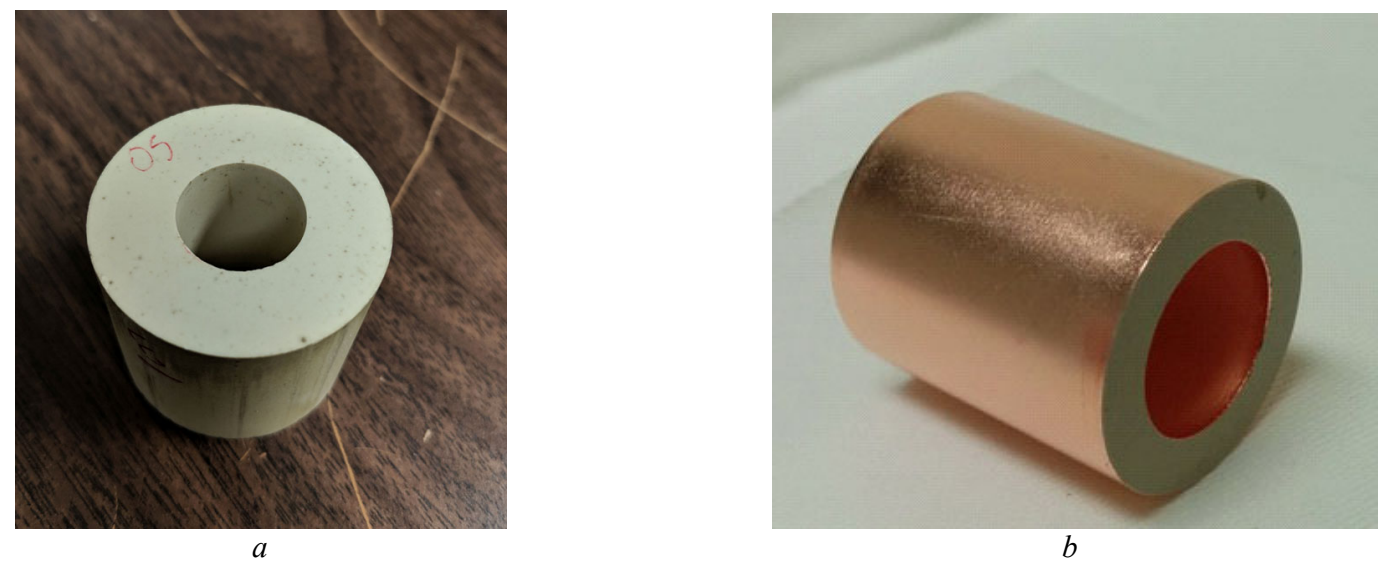

Fig. 3. The tunable BST ferroelectric element for $400 \mathrm{MHz}$ SRF cavity tuner (a); tunable ferroelectric BST element after metallization $(b)$

sions of low permittivity conventional dielectric material. In particular, the area of possible accelerator applications of ferroelectric elements is broadening. Our collaboration currently has studied two L-band tuner designs, and X-band and Ka-band fast switches and phase shifters $[12,13]$. A fast ferroelectric tuner has been designed for operation in L-band to allow rapid adjustments of cavity coupling in an accelerator where RF source fluctuations, microphonics, or other uncontrolled fluctuations could cause undesirable emittance growth. This ferroelectric based device is intended for ERL [11], ILC and PIP-II applications [12, 13].

Typical representative ferroelectric materials are $\mathrm{BaTiO}_{3}$ or a $\mathrm{BaTiO}_{3}-\mathrm{SrTiO}_{3}$ solid solution (BST). The BST solid solution can be synthesized in the form of polycrystalline ceramic layers and in bulk, Fig. 2, b. BST(M) material [26] (BST ferroelectric with Mg-based additives) was developed by Euclid Techlabs/Ceramic Ltd. collaboration allowing fast switching and tuning in vacuum at a high biasing electric field of $50 \mathrm{kV} / \mathrm{cm}$ [27, 28]. Initially this material was developed for the $\mathrm{X}$-band frequency range $[29,30](11.424 \mathrm{GHz})$ and demonstrated loss tangents of $5 \cdot 10^{-3}$ at $10 \mathrm{GHz}$. Tunability, time response and loss factor measurements for large bulk ferroelectric samples have been presented and published in [31]. A new BST-based material has been developed with a tunability of $6-8 \%$ at a $15 \mathrm{kV} / \mathrm{cm}$ biasing field to be applied in air [32-34]. Development of this type of material was a challenge; there are no other materials available with a tuning range and loss factor close to those listed above. It was demonstrated recently that by introducing a linear (non-tunable)
Mg-based ceramic component into the BST solid solution one can enhance the tunability factor of the entire composition while keeping the loss tangent below $10^{-3}$ at $\mathrm{L}$ band [33-34]. This counter-intuitive property (by increasing the non-tunable ceramic content of the ferroelectric-ceramic mixture one can enhance the tunability of the resulting material) opens important new possibilities in designing the specific class of microwave ceramic materials that will enable tuning at low magnitude biasing fields. In particular, an unprecedented low zero-field permittivity, non-linear material that retains tunability has been developed [33-34]: a BST ferroelectric and $\mathrm{Mg}$ based additive composite with a dielectric constant in the range of $\approx 150$, Fig. 3, $a, b$.

A prototype Ferroelectric Fast Reactive Tuner (FE-FRT) for superconducting cavities has been developed, which allows the frequency to be controlled by application of a potential difference across a ferroelectric residing within the tuner. This technique has now become practically feasible due to the recent development of a new extremely low loss ferroelectric material. In a world first, CERN has tested the prototype FE-FRT (based on the Euclid/Ceramics Ltd. BST material) with a superconducting cavity, and frequency tuning has been successfully demonstrated [11]. This is a significant first step in the development of an entirely new class of tuner. These will allow electronic control of cavity frequencies, by a device operating at room temperature, within timescales that will allow active compensation of microphonics. For many applications this could eliminate the need to use over-coupled fundamental power 
couplers, thus significantly reducing RF amplifier power. Details of this research can be found in [11].

Conclusion. We presented a survey of experimental testing of microwave materials with highcurrent electron beams and high power microwave sources intended for use in dielectric wakefield acceleration and other beam physics applications. The discussed materials include low loss microwave ceramics, quartz (fused silica), CVD diamonds and nonlinear BST based ferroelectrics. We presented our experimental results on wakefield generation in microwave frequency ranges with the dielectric based accelerating structures. Special attention was paid to the experimental results on high power testing at Xband for the externally powered dielectric based structures. Finally, we presented here first experimental demonstration of application of ferroelectric tunable microwave ceramic for accelerator application, which includes both tunable dielectric wakefield accelerating structures and ferroelectric based fast high power tuner for superconducting cavities. Experimental results presented here are critically important for the advanced dielectric wakefield accelerating structures and other components development intended for the future linear collider projects.

\section{References}

1. Gai W., Schoessow P., Cole B., Konecny R., Norem J., Rosenzweig J., Simpson J. Experimental Demonstration of Wake-Field Effects in Dielectric Structures. Phys. Rev. Lett. 1988, vol. 61, 2756. doi: 10.1103/PhysRevLett.61.2756

2. Gai W., Conde M., Power J. G., Jing C. Considerations for a Dielectric-based Two-beam-accelerator Linear Collider. Proc. Int. Particle Accelerator Conf. IPAC'10, Kyoto, Japan, 2010, pp. 3428-3430.

3. Gai W. Advanced Accelerating Structures and Their Interaction with Electron Beams. AIP Conf. Proc., American Institute of Physics, New York, 2009, vol. 1086, pp. 3-11. doi: 10.1063/1.3080940

4. Conde M. Survey of Advanced Dielectric Wakefield Accelerators. Proc. Part. Accelerator. Conf. PAC'07, Albuquerque, New Mexico, USA. July 2007, pp. 1899-1903.

5. Kanareykin A. New Advanced Dielectric Materials for Accelerator Applications. AIP Conf. Proc., American Institute of Physics, New York, 2010, vol. 1299, pp. 286291. doi: 10.1063/1.3520329

6. Kanareykin A. Cherenkov radiation and dielectric based accelerating structures: wakefield generation, power extraction and energy transfer efficiency. Journal of Physics: Conf. Series, 2010, vol. 236, p. 012032.

7. Thompson M. C., Badakov H., Rosenzweig J. B., Travis G., Hogan M., Ischebeck R., Kirby N., Siemann R., Walz D., Muggli P., Scott A., Yoder R. Ultra-High Gradient Dielectric Wakefield Accelerator Experiments. AIP Conf. Proc., American Institute of Physics, New York, 2006, vol. 877, pp. 903-909. doi: 10.1063/1.2409232

8. Whittum D. H. Ultimate Gradient in Solid-State Accelerators. Preprint SLAC-PUB-7910, July 1998. Available at: https://www.slac.stanford.edu/pubs/slacpubs/7750/slacpub-7910.pdf (accessed 15.08.2019)

9. Wang C., Yakovlev V. P., Hirshfield J. L. Rectangular Diamond-Lined Accelerator Structure. Proc. Particle Accelerator Conf. PAC'05, 2005, pp. 1282-1285. doi: 10.1109/PAC.2005.1590735

10. Schoessow P., Kanareykin A., Gat R. CVD Diamond Dielectric Accelerating Structures. AIP Conf. Proc.,
American Institute of Physics, New York, 2009, vol. 1086, pp. 398-403. doi: 10.1063/1.3080939

11. Kazakov S., Shchelkunov S., Yakovlev V., Kanareykin A., Nenasheva E., Hirshfield J. L. Fast ferroelectric phase shifters for energy recovery linacs. Physical Review. ST-Accelerator and Beams, 2010, vol. 13, 113501. doi: 10.1103/PhysRevSTAB.13.113501

12. Kanareykin A., Jing C., Nenasheva E., Schoessow P., Power J. G., Gai W. Development of a Ferroelectric Based Tunable DLA Structure. AIP Conf. Proc., American Institute of Physics, New York, 2009, vol. 1086, pp. 386-392. doi: 10.1063/1.3080936

13. Sotnikov G. V., Marshall T. C., Shchelkunov S. V., Didenko A., Hirshfield J. L. Two-Channel Rectangular Dielectric Wake Field Accelerator Structure Experiment. AIP Conf. Proc., 2009, vol. 1086, p. 415. doi: 10.1063/1.3080943

14. Kanareykin A., Nenasheva E., Kazakov S., Kozyrev A., Tagantsev A., Yakovlev V., Jing C. Ferroelectric Based Technologies for Accelerators. AIP Conf. Proc., American Institute of Physics, New York, 2009, vol. 1086, pp. 380-385. doi: 10.1063/1.3080935

15. Gai W., Conde M. E., Konecny R., Power J. G., Schoessow P., Zou P. RF power generation and coupling measurements for the dielectric wakefield step-up transformer. AIP Conf. Proc., American Institute of Physics, New York, 1999, vol. 472, pp. 626-634.

16. Gao F., Conde M. E., Gai W., Jing C., Konecny R., Liu W., Power J. G., Wong T., Yusof Z. Design and testing of a $7.8 \mathrm{GHz}$ power extractor using a cylindrical dielectric-loaded waveguide. Physical Review. ST-Accelerator Beams, 2008, vol. 11, p. 041301-1. doi: 10.1103/PhysRevSTAB.11.041301

17. Jing C., Liu W., Gai W., Power J., Wong T. Mode analysis of a multilayered dielectric-loaded accelerating structure. Nuclear Instruments and Methods in Physics Research Section A, 2005, vol. 539, iss. 3, pp. 445-454. doi: 10.1016/j.nima.2004.10.030

18. Jing C., Kanareykin A., Kazakov S., Liu W., Nenasheva E., Schoessow P., Gai W. Development of a dual- 
layered dielectric-loaded accelerating structure. Nuclear Instruments and Methods, A, 2008, vol. 594, pp. 132-139.

19. Zou P., Gai W., Konecny R., Kanareykin A., Konecny R., Sun X., Wong T. Construction and Testing of an $11.4 \mathrm{GHz}$ Dielectric Structure Based Traveling Wave Accelerator. Review of Scientific Instruments, 2000, vol. 71, p. 2301. doi: 10.1063/1.1150446

20. Jing C., Kanareykin A., Power J. G., Conde M., Yusof Z., Schoessow P., Gai W. Observation of Enhanced Transformer Ratio in Collinear Wakefield Acceleration. Phys. Rev. Lett., 2007, vol. 98, p. 144801 . doi: 10.1103/PhysRevLett.98.144801

21. Jing C., Kanareykin A., Schoessow P., Conde M. E., Gai W., Konecny R., Power J. G. The First Experiment of a $26 \mathrm{GHz}$ Dielectric Based Wakefield Power Extractor. Proc. Int. Particle Accelerator Conf. IPAC'10, Kyoto, Japan, 2010, pp. 4434-4436.

22. Yu D., Newsham D., Smirnov A. V., Gai W., Konecny R., Liu W., Braun H., Carron G., Döbert S., Thorndahl L., Wilson I., Wuensch W. Construction and Testing of a $21 \mathrm{GHz}$ Ceramic Based Power Extractor. Proc. Particle. Accelerator Conf. PAC'03, 2003, p. 11561159. doi: 10.1109/PAC.2003.1289637

23. Jing C., Gai W., Power J. G., Konecny R., Liu W., Gold S. H., Kinkead A. K., Tantawi S. G., Dolgashev V., Kanareykin A. Progress Toward Externally Powered XBand Dielectric-Loaded Accelerating Structures. IEEE Trans. Plasma Science, 2010, vol. 38, iss. 6, pp. 13541360. doi: 10.1109/TPS.2009.2036921

24. Jing C., Gai W., Power J. G., Konecny R., Gold S. H., Liu W., Kinkead A. K. High-power RF tests on X-band dielectric-loaded accelerating structures. IEEE Trans. on Plasma Science. 2005, vol. 33, iss. 4, pp. 1155-1160. doi: 10.1109/TPS.2005.851957

25. Power J. G., Conde M. E., Gai W., Konecny R., Schoessow P., Kanareykin A. Measurements of the longitudinal wakefields in a multimode, dielectric wakefield a accelerator driven by a train of electron bunches. Physical Review. ST-Accelerator and Beams. 2000, vol. 3, iss. 10, 101302. doi: 10.1103/PhysRevSTAB.3.101302

26. Shipman N. C., Coly M. R., Ben-Zvi I., Bastard J., Gerigk F., Macpherson A., Stapley N., Nenasheva E., Jing C.-J., Kanareykin A., Kazakov S., Burt G., Castilla A. A Ferroelectric Fast Reactive Tuner for Superconducting Cavities. Proc. of the Superconducting RF Workshop, SRF'2019, Dresden, Germany, 5-9 July 2019.
27. Nenasheva E., Kanareikin A. Low Dielectric Loss Ceramic Ferroelectric Composite Material. 2011. US Patent № 8067324.

28. Nenasheva E. A., Kanareykin A. D., Kartenкo N. F., Karmanenко S. F. Ceramics Materials Based on $(\mathrm{Ba}, \mathrm{Sr}) \mathrm{TiO}_{3}$ Solid Solutions for Tunable Microwave Devices. Journal of Electroceramics. 2004, vol. 13, pp. 235-238.

29. Kanareykin A., Kazakov S., Nenasheva E., Tagantsev A., Yakovlev V. P. Tunable Ferroelectric Based Technologies for Accelerator Components. Proc. of European Particle Accelerator Conf. EPAC 2008. Genoa, Italy, 2008, pp. 1646-1648.

30. Yakovlev V. P., Nezhevenko O. A., Hirshfield J. L., Kanareykin A. D. Ferroelectric Switch for an Active RF Pulse Compressor. AIP Conf. Proc. American Institute of Physics, New York, 2003, vol. 691, pp. 187-196. doi: 10.1063/1.1635119

31. Jing C., Kanareykin A., Power J. G., Conde M., Liu W., Antipov S., Schoessow P., Gai W. Experimental Demonstration of Wakefield Acceleration in a Tunable Dielectric Loaded Accelerating Structure. Phys. Rev. Lett. 2011, vol. 106, pp. 164802. doi: 10.1103/PhysRevLett.106.164802

32. Kazakov S. Yu., Shchelkunov S. V., Yakovlev V. P., Kanareykin A., Nenasheva E., Hirshfield J. L., Khabiboulline T., Hahn H., Choi E. M. Fast High-Power Microwave Ferroelectric Phase Shifters for Accelerator Application. AIP Conf. Proc. American Institute of Physics, New York, 2009, vol. 1086, pp. 477-484. doi: 10.1063/1.3080953

33. Nenasheva E. A., Kartenko N. F., Gaidamaka I. M., Trubitsyna O. N., Kanareykin A. D. Low loss Microwave Ferroelectric Ceramics for High Power Tunable Devices. Journal of European Ceramic Society. 2010, vol. 30, pp. 395-400. doi: 10.1016/j.jeurceramsoc.2009.04.008

34. Kozyrev A. B., Kanareykin A. D., Nenasheva E. A., Osadchy V. N., Kosmin D. M. Observation of an anomalous correlation between permittivity and tunability of a doped $(\mathrm{Ba}, \mathrm{Sr}) \mathrm{TiO}_{3}$ ferroelectric ceramic developed for microwave applications. Applied Physics Letters. 2009, vol. 95, iss. 1, pp. 012908-012910. doi: 10.1063/1.3168650

35. Kanareykin A., Kazakov S., Kozyrev A. B., Nenasheva E., Yakovlev V. P. Observation of an Anomalous Tuning Range of a Doped BST Ferroelectric Material Developed for Accelerator Applications. Proc. of Int. Particle Accelerator Conf. IPAC 2010, Kyoto, Japan, 2010, pp. 3987-3989.

\section{Information about the author}

Alexei D. Kanareykin - Dr. Sci. (Physics) of St Petersburg State University (2012), President and CEO of Euclid Techlabs LLC, Solon, OH USA. The author of more than 300 scientific publications. Area of expertise: beam physics, advanced methods of acceleration, dielectric and ferroelectric materials at extreme conditions.

E-mail: alex.kanareykin@gmail.com 